\title{
Survival Probability in a Random Velocity Field
}

\author{
S. Redner \\ Center for Polymer Studies and Department of Physics, Boston University, Boston, MA, 02215
}

\begin{abstract}
The time dependence of the survival probability, $S(t)$, is determined for diffusing particles in two dimensions which are also driven by a random unidirectional zero-mean velocity field, $v_{x}(y)$. For a semi-infinite system with unbounded $y$ and $x>0$, and with particle absorption at $x=0$, a qualitative argument is presented which indicates that $S(t) \sim t^{-1 / 4}$. This prediction is supported by numerical simulations. A heuristic argument is also given which suggests that the longitudinal probability distribution of the surviving particles has the scaling form $P(x, t) \sim t^{-1} u^{1 / 3} g(u)$. Here the scaling variable $u \propto x / t^{3 / 4}$, so that the overall time dependence of $P(x, t)$ is proportional to $t^{-5 / 4}$, and the scaling function $g(u)$ has the limiting dependences $g(u) \propto$ const. as $u \rightarrow 0$ and $g(u) \sim \exp \left(-u^{4 / 3}\right)$ as $u \rightarrow \infty$. This argument also suggests an effective continuum equation of motion for the infinite system which reproduces the correct asymptotic longitudinal probability distribution.
\end{abstract}

PACS Numbers: 05.40.+j, 05.60.+w, 02.50.Ey

\section{INTRODUCTION}

Consider a diffusing particle in the semi-infinite planar domain $(x>0, y)$ which is absorbed when $x=0$ is reached. In addition to the diffusion, the particle is driven by a unidirectional random velocity field in which $v_{x}(y)$ is a random, zero-mean function of $y$ only (Fig. 1). This type of stochastic motion was introduced by Matheron and de Marsily (MdM) [1] to describe the hydrodynamic dispersion of dynamically-neutral tracer in a sedimentary layered rock formation. Although the longitudinal bias averaged over an infinite number of transverse layers is zero, the typical bias over a finite number of layers is a fluctuating quantity which is a decreasing function of the number of layers that a random walk visits. This non-vanishing residual bias underlies the faster than diffusive transport of the model. In an infinite system it has been established that the typical horizontal

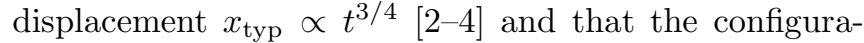
tion averaged distribution of longitudinal displacements has the form $P(x, t) \sim t^{-3 / 4} \exp \left[-\left(x / t^{3 / 4}\right)^{4 / 3}\right]$. There are also strong fluctuations in the probability distribution between different samples of the velocity field, as well as a very slow convergence to the asymptotic limit.

While much is now understood about transport in the MdM model, we wish to investigate its first passage properties. Specifically, we consider the semi-infinite twodimensional system $x>0$ with an absorbing boundary at $x=0$, and study the time dependence of the particle survival probability, $S(t)$. The survival probability in a finite system with absorbing boundaries at $x= \pm L$ and the same unidirectional random velocity field has been studied previously [3]; however this system exhibits fundamentally different behavior than the semi-infinite system that is treated here.

In the absence of a velocity field, it is well known that in the semi-infinite system $S(t)$ asymptotically decays in time as $t^{-1 / 2}$ [5. Because the velocity field in the MdM model has no net longitudinal bias, it is not immediately obvious how the behavior of $S(t)$ will be affected. Naively, one might expect that the dominant contribution to $S(t)$ will arise from those velocity configurations whose average bias is directed away from the boundary. This is indeed the case, and from this starting point, we present a simple argument which suggests that the survival probability, averaged over all realization of the velocity field, is proportional to $t^{-1 / 4}$. This prediction is in excellent agreement with our numerical results.

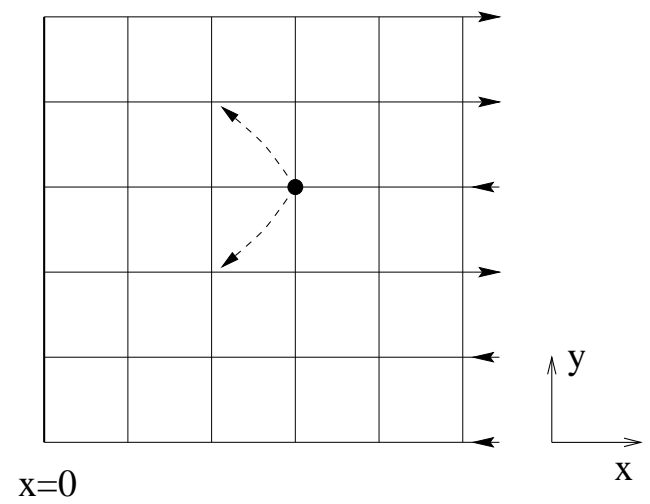

FIG. 1. The random velocity field in a realization of the MdM model on a finite width square lattice strip. On the horizontal bonds, the direction of the velocity field is indicated by the arrows. In a single time step, a particle (heavy dot) can move equiprobably only to one of the two target sites indicated. Particles are absorbed at $x=0$.

Interestingly, a $t^{-1 / 4}$ decay of the survival probability has been found previously for diffusing particles in a semi-infinite two dimensional system with a unidirectional zero-bias, but deterministic velocity field of the form $v_{x}(y)=-v_{x}(-y)$ [6]. Although the mechanism that leads to $S(t) \sim t^{-1 / 4}$ in this class of velocity fields is different than that for the MdM model, the two systems 
share the feature that their velocity fields have no average bias in $x$. It would be interesting to determine whether a $t^{-1 / 4}$ decay of the survival probability is characteristic of all semi-infinite systems with a zero-mean longitudinal velocity field.

In the next section, we give a heuristic argument which suggests that $S(t) \propto t^{-1 / 4}$. We then present corroborating numerical simulations in Sec. III. We also find that the spatial probability distribution of the surviving particles provides insight into the formulation of a continuum equation of motion for the longitudinal probability distribution in an unbounded geometry. Thus in Sec. IV, we infer this equation of motion and, from its solution, determine the correct asymptotic longitudinal probability distribution in the unbounded geometry. We conclude with a brief discussion in Sec. V.

\section{CONFIGURATION-AVERAGED SURVIVAL PROBABILITY}

We first present our argument for the the time dependence of $S(t)$. The basic idea is that the dominant contribution to this average arises from the subset of all velocity configurations whose net bias is away from the boundary, $i$. e., in the $+x$-direction. Conversely, configurations with a bias along $-x$ will give individual contributions to $S(t)$ which decay exponentially in time and thus should be asymptotically negligible.

To determine which of the positively-biased velocity configurations give the dominant contribution to $S(t)$, consider a discrete realization of the MdM model on the square lattice in which the velocity is either $+v_{0}$ or $-v_{0}$ with equal probability for a given value of $y$ (Fig. 1). Periodic boundary conditions in the transverse direction are employed, so that the system is a semi-infinite cylinder consisting of $w$ rows. For concreteness, the initial condition is $p(x, y, t=0)=\frac{1}{w} \delta_{x, \ell}, i$. e., a ring of particles is initially placed at $x=\ell$, where $\ell$ is the lattice spacing. In this system, the probability that there are $n_{+}$ positively biased rows and $n_{-}$negatively biased rows is $\mathcal{P}(m) \propto \frac{1}{\sqrt{w}} e^{-m^{2} / w}$, with $m=n_{+}-n_{-}$. In a time $t$, the number of layers visited by a random walk is $w \propto \sqrt{D t} / \ell$, where $D$ is the transverse (microscopic) diffusivity. By transforming from $m$ to the velocity $v=m v_{0} / w$, the distribution of velocities within $w$ layers is

$$
\mathcal{P}(v) \propto \frac{1}{v_{0}}\left(\frac{D t}{\ell^{2}}\right)^{1 / 4} \exp \left[-\left(\frac{v}{v_{0}}\right)^{2}\left(\frac{D t}{\ell^{2}}\right)^{1 / 2}\right] .
$$

Because this distribution is strongly cut off when the argument of the exponential is greater than one, we expect that the dominant contribution to $S(t)$ will arise from those velocity configurations whose net bias is within the range $0<v<v_{0}\left(\ell^{2} / D t\right)^{1 / 4}$.

For a positively biased velocity configuration, we now estimate the residual survival probability in the long time limit under the assumption that $w$ is finite. In this case, the particle will uniformly sample the transverse extent of the system and it is sensible to characterize the bias by its mean value $v$. If $v$ is small, or more properly the Peclet number $v x_{0} / D$ is small, then for $t<D / v^{2}$ the bias is irrelevant and consequently $S(t) \sim x_{0} / \sqrt{D t}$, where $x_{0}$ is the initial position of the particle [5]. However, for $t>D / v^{2}$, convection dominates and returns to the origin become extremely unlikely. Hence $S(t)$ should "stick" at the value attained when $t=D / v^{2}$. This implies that the asymptotic behavior of the survival probability is simply, $S(t=\infty) \propto v x_{0} / D$. This same result can also be obtained with additional effort from a more rigorous approach in which one solves the onedimensional convection-diffusion equation on the domain $x>0$ with the initial condition $P(x, t=0)=\delta\left(x-x_{0}\right)$ and then computes $S(t)$ by integrating this probability density over all $x>0$.

For the MdM model, we now average over all relevant velocity configurations to determine $S(t)$. That is

$$
\begin{aligned}
S(t) & \sim \int_{0}^{\infty} \frac{v x_{0}}{D} \frac{1}{v_{0}}\left(\frac{D t}{\ell^{2}}\right)^{1 / 4} \exp \left[-\left(\frac{v}{v_{0}}\right)^{2}\left(\frac{D t}{\ell^{2}}\right)^{1 / 2}\right] d v \\
& =\frac{1}{2} \frac{v_{0} x_{0}}{D}\left(\frac{\ell^{2}}{D t}\right)^{1 / 4} \\
& \propto t^{-1 / 4}
\end{aligned}
$$

\section{NUMERICAL SIMULATIONS}

We now present numerical evidence to support the prediction that $S(t) \propto t^{-1 / 4}$. For simulating the particle motion, we propagate the probability distribution for each velocity configuration exactly $[7]$. The microscopic motion is defined by the rule that in a single time step, a particle hops equiprobably by $\pm \ell$ in the $y$-direction and by a distance $\operatorname{sign}(v(y)) \ell$ in the $x$-direction on the square lattice, where $v(y)$ is the velocity field at the $y$-coordinate of the particle before the hopping event (Fig. 1). Accordingly, the probability that a particle is at $(x, y)$ at time $t$ evolves according to

$$
\begin{aligned}
p(x, y, t+1) & =\frac{1}{2} p(x-\ell \times \operatorname{sign}(v(y-\ell)), y-\ell, t) \\
& +\frac{1}{2} p(x-\ell \times \operatorname{sign}(v(y+\ell)), y+\ell, t) .
\end{aligned}
$$

For each velocity configuration, probability propagation yields the exact distribution in the presence of the absorbing boundary up to the maximum time specified.

We have performed the average over velocity configurations in two complementary ways - either an average over a representative sample of velocity configurations, or an exhaustive average over all velocity configurations for relatively small systems. The former is straightforward to implement, but the effect of rare configurations on the results is unknown. The latter, on the other hand, 
gives the exact result for an infinite system, albeit only for short times. This exactness allows one to test for systematic trends in the data, an analysis which is not feasible when averaging over a representative set of velocity configurations.

A typical result for $S(t)$ up to $t=4095$, based on an average over 50 velocity configurations on a cylinder of width 400, is shown in Fig. 2. The average bias of these 50 configurations turns out to be -0.004 . Beyond approximately 50 time steps, the data for the survival probability is quite linear and a least-squares power law fit to the data in this time range yields the exponent of -0.2491 . Further, the slope between successive data points, or local exponent estimate

$$
\alpha_{w}(t) \equiv \frac{\ln (S(t) / S(t-1))}{\ln (t /(t-1))}
$$

deviates from -0.25 by less than 0.002 for $t \gtrsim 50$ (inset). To check that finite width effects do not substantially affect the results, we also considered shorter times, $t \lesssim 1600$ and a slightly narrower system $(w=300)$, and averaged over 250 realizations. The average bias of these configurations turns out to be +0.002 . This case yielded a best fit exponent of -0.2503 . These two data sets strongly suggest that $S(t) \propto t^{-1 / 4}$ in the long time limit. However, because the average is performed only over an infinitesimal fraction of all velocity configurations, it is possible that extreme configurations could alter the results. For this reason, we now investigate the exact behavior of $S(t)$ for short times by averaging over all velocity configurations.

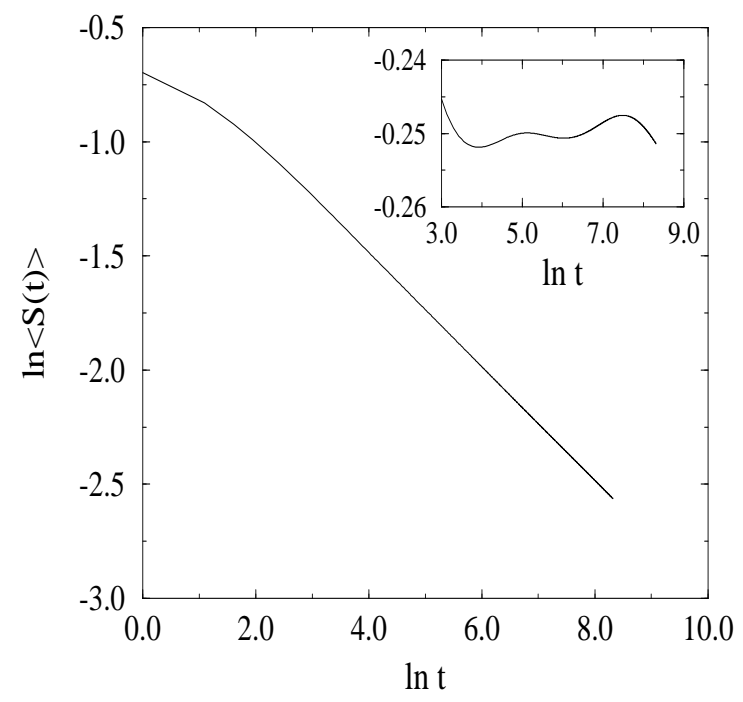

FIG. 2. Plot of $S(t)$ versus $t$ on a double logarithmic scale, based on averaging the exact probability distribution over a finite number of velocity configurations for the system discussed in the text. The inset shows the local slopes between neighboring data points.
In this complete enumeration, we consider odd values of $w$ for convenience. Because of the periodic transverse boundary conditions, many of the $2^{w}$ configurations are identical up to cyclic permutation and reflection symmetry. To carry out the enumeration, we first encode each velocity configuration as a binary sequence. Using bit manipulation techniques, we identify the "irreducible" representation of this binary sequence, defined as the smallest equivalent integer number obtained by performing all possible cyclic permutations of the initial binary sequence. This same procedure is then repeated on the reversed initial binary sequence. Thus to each binary sequence there is a unique irreducible binary sequence. By this mapping, we only need consider the irreducible configurations and weight each by their degeneracy in performing the average over velocity configurations. For example for $w=23,25,27$, and 29, the number of irreducible configurations are $92,205,337,594,1,246863$, and $4,636,390$, compared, e. g., to $2^{29}=536,870,912$. For each irreducible configuration, we then perform the exact probability distribution propagation. This complete enumeration provides the exact value of $S(t)$ for an infinite system up to $w-1$ time steps, while finite width crossover effects gradually begin to play a role for later times.

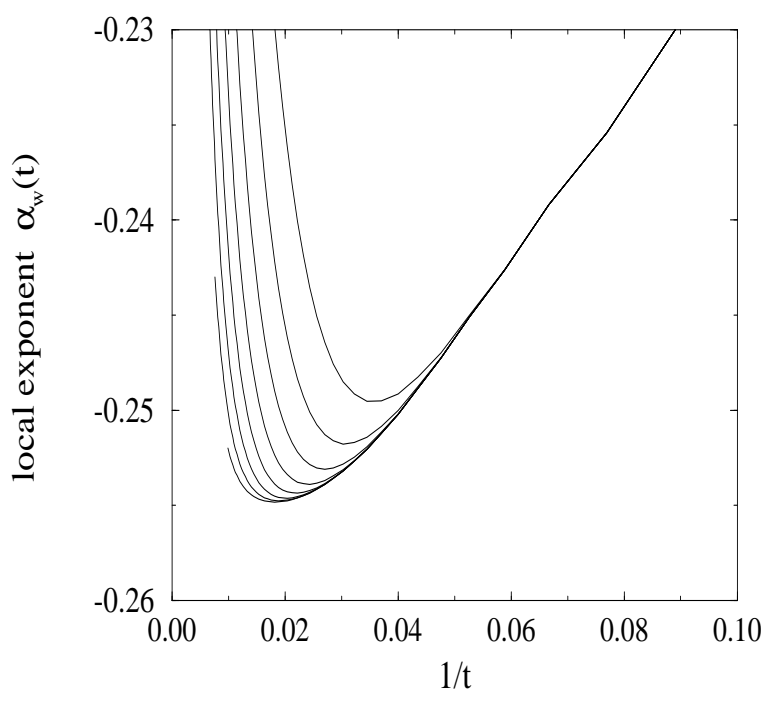

FIG. 3. The local slope of $S(t)$ versus $t$ on a double logarithmic scale, based on averaging the exact probability distribution over all of velocity configurations for systems of width $w=15,17, \ldots, 29$. The data for different $w$ coincide for larger $1 / t$, but then separate at progressively smaller $1 / t$ as $w$ increases

We therefore typically carried out the probability propagation for up to $t \approx 2 w$ time steps and exploited the crossover in $S(t)$ to interpret our results. A plot of $\ln S(t)$ versus $\ln t$ should initially show power law behavior, indicative of the infinite system behavior, and then cross 
over to a non-zero constant because of finite width effects. Thus a plot of the local exponent $\alpha_{w}(t)$ (here defined as the slope between every other data point) versus $1 / t$ should initially provide an estimate of the exponent of $S(t)$, while the crossover effect determines the time range over which the exact data is relevant for the infinite system. In Fig. 3, this local slope, is plotted versus $1 / t$ for system widths $w$ between 15 and 29. Initially, $\alpha_{w}(t)$ is decreasing nearly linearly in $1 / t$, but subsequently there is the expected crossover to the asymptotic value of zero. In the regime where the data is relatively linear, we compute the intercepts of successive data points at $1 / t=0$ as an estimate of the asymptotic value of the exponent (Fig. 4). As $w$ increases, this data exhibits: (i) nonmonotonic trends in the data (e.g., the location of the minimum) which disappear only for $w \geq 25$, (ii) more stable extrapolated values as $w$ increases, and (iii) the minimum value of the extrapolated exponent - which we adopt as the best estimate of the exponent for a given value of $w$ - is increasing systematically in $w$ and appears to be converging to -0.25 . While there is slow convergence to asymptotic behavior which gives rise to considerable subjectivity in analysis, we believe that the trends in the data support the hypothesis that $S(t) \sim t^{-1 / 4}$.

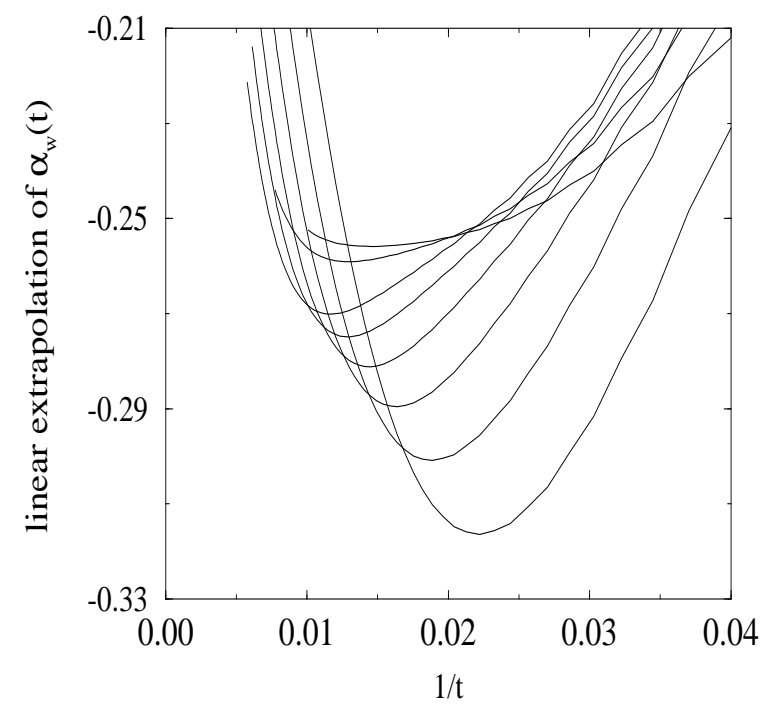

FIG. 4. Linear extrapolation of the local slope from Fig. 3 for $w=15,17, \ldots, 29$. The minimum value is progressively increasing with $w$.

\section{THE PROBABILITY DISTRIBUTION}

In addition to investigating $S(t)$, we also examined the probability distribution of the surviving particles. This quantity provides an alternative understanding for the first-passage process, as well as useful fundamental insights about the continuum description of MdM model. Specifically, we study $P(x, t) \equiv \int p(x, y, t) d y$, the con- figuration averaged longitudinal probability distribution of particles which have not yet been absorbed by time $t$. We expect that this probability distribution can be written in a scaling form

$$
P(x, t)=A f(x /\langle x\rangle),
$$

where $\langle x\rangle=\langle x(t)\rangle$ is the average longitudinal displacement of the survivors at time $t$. Monte Carlo simulations clearly indicate that $\langle x\rangle \propto t^{3 / 4}$, as in the case when there is no absorbing boundary present 杖国. Because $\int_{0}^{\infty} P(x, t) d x=S(t)$, we can determine the coefficient $A$ by integrating Eq. (5) over $x$ and thereby write

$$
P(x, t)=\frac{S(t)}{\langle x\rangle F_{0}} f(x /\langle x\rangle),
$$

where $F_{0}=\int_{0}^{\infty} d u f(u)$. Because of the absorbing boundary, $P(x=0, t)$ must equal zero, leading to the expectation that $f(u)$ will vanish as a power law as $u \rightarrow 0$. Consequently, we write

$$
P(x, t)=\frac{S(t)}{\langle x\rangle F_{0}}\left(\frac{x}{\langle x\rangle}\right)^{\mu} g(x /\langle x\rangle),
$$

with $g(u) \rightarrow$ const. as $u \rightarrow 0$, and $g(u)$ vanishing faster than any power law for $u \rightarrow \infty$.

A plot of the scaling function $f(u)$ versus $u$ is shown in Fig. 4 for $t=255,1023$, and 4095. There is a small but systematic variation in the data for different times, with the small- $u$ behavior steepening and the large- $u$ tail growing for larger time. Nevertheless, reasonable data collapse is obtained in which $f(u)$ qualitatively exhibits the expected power-law and rapid cutoff asymptotic behaviors for small and large $u$, respectively. We attribute the small deviation from scaling on slow convergence to the asymptotic limit (see below). Such a phenomenon was observed previously in the probability distribution for an infinite system [2,3, and similar slow convergence effects can be anticipated here as well.

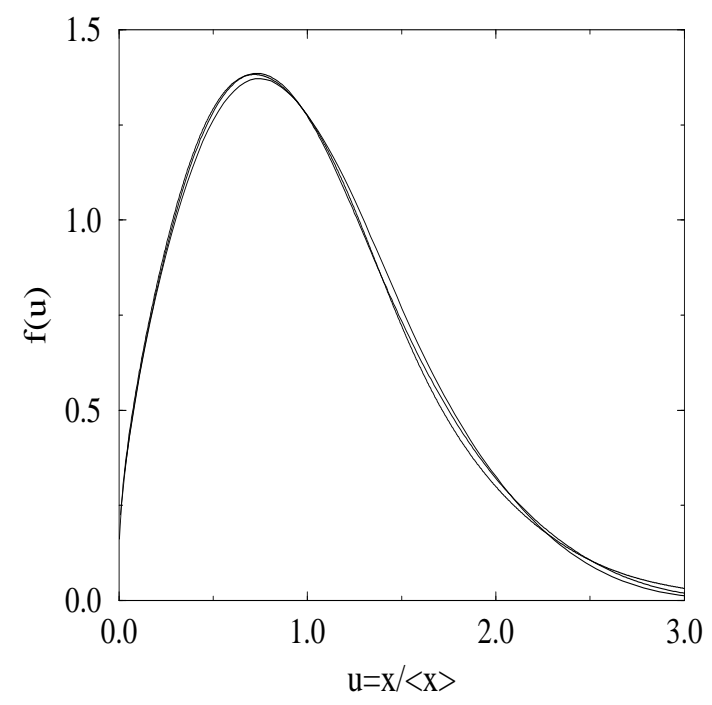


FIG. 5. The scaling function $f(u)$ versus $u$.

The exponent $\mu$ in Eq. (7) can be obtained by demanding consistency between the time dependence of $S(t)$ and that of the first-passage probability. If $S(t) \propto t^{-1 / 4}$, then from the general relation [5] between $S(t)$ and the first passage probability to the boundary, $\mathcal{F}(t)$, we have $\frac{\mathcal{F}(t)=-d S(t)}{d t \propto t^{-5 / 4}}$. On the other hand for a normalized initial condition, the first passage probability coincides with the flux to $x=0$. Now as $x \rightarrow 0$, Eq. (7) gives

$$
P(x, t) \propto \frac{S(t)}{\langle x\rangle^{1+\mu}} x^{\mu} \propto t^{-1 / 4-3(1+\mu) / 4} x^{\mu} .
$$

Since the flux is obtained by performing an appropriate spatial derivative of this limiting probability distribution, an operation which does not affect the temporal behavior, we conclude that $\mu=1 / 3$ to recover the correct $t^{-5 / 4}$ time dependence for the flux.

However, the data in Fig. 5 does not exhibit this behavior because of finite time effects. For small $u$, the numerical value of $g(u)$ at $x=1$ is non-zero but decreasing with time. Correspondingly, the value of $u$ at this first data point is non-zero but also decreasing with time. This anomaly in the small- $u$ data renders a simple power law fit inadequate. However, such a naive fit to the data in the range $u<1 / 2$ gives the estimates 0.483 , $0.479,0.476,0.470$, and 0.464 , respectively, for the exponent $\mu$ in $P(x, t)$ for the 5 aforementioned time values. Other analyses, such as computing the first derivative of $f(u)$ (which should diverge as $u^{-2 / 3}$ ) and determining the exponent of the small- $u$ dependence, lead to a similar quantitative conclusion. While the anticipated value $\mu=1 / 3$ is not obtained, we believe that better agreement with theory would emerge if it were practical to extend our Monte Carlo simulations to much longer times.

The existence of this power-law prefactor further suggests that in a continuum description, the configuration averaged flux can be obtained via

$$
\mathcal{F}(t) \propto-\left.\frac{\partial P(x, t)}{\partial x^{1 / 3}}\right|_{x=0} \propto-\left.x^{2 / 3} \frac{\partial P(x, t)}{\partial x}\right|_{x=0} .
$$

Such a scale-dependent diffusivity can be heuristically justified by appealing to a Taylor diffusion description of the motion in the MdM model [8]. In a time $t$, a particle typically explores $w \approx \sqrt{D t}$ transverse layers. The typical bias within this number of layers is then proportional to $w^{-1 / 2}$ or $(D t)^{-1 / 4}$. Thus in a time scale $t$, the typical longitudinal distance travelled by a particle is $d \sim v t \propto t^{3 / 4}$. Because these segments of length $d$ are randomly in the $+x$ or $-x$ direction and the time interval between segments is of order $t$, we infer an effective longitudinal diffusion (or dispersion) coefficient $D_{\|} \sim \ell^{2} / t \propto t^{1 / 2} \propto x^{2 / 3}$, as written in Eq. (9).

Let us now pursue the consequences of this scale dependent diffusion coefficient for the longitudinal motion. If the longitudinal flux $j(x, t)$ is indeed proportional to $\frac{-x^{2 / 3} \partial P(x, t)}{\partial x}$, then substituting this into the continuity equation

$$
\frac{\partial P(x, t)}{\partial t}+\frac{\partial j(x, t)}{\partial x}=0,
$$

leads to the effective equation of motion

$$
\frac{\partial P(x, t)}{\partial t}=\frac{\partial}{\partial x} x^{2 / 3} \frac{\partial P(x, t)}{\partial x} .
$$

We can easily solve this equation by applying scaling. Assuming that $P(x, t) \propto t^{-3 / 4} h\left(x / t^{3 / 4}\right)$, we rewrite the partial derivatives in $x$ and $t$ in terms of a derivative with respect to $u \equiv x / t^{3 / 4}$, to recast the equation of motion as

$$
-\frac{3}{4}(u h(u))^{\prime}=\left(u^{2 / 3} h^{\prime}(u)\right)^{\prime} .
$$

Here the prime denotes differentiation with respect to $u$. One integration immediately yields

$$
-\frac{3}{4} u h(u)=\left(u^{2 / 3} h^{\prime}(u)\right) .
$$

The constant of integration equals zero because $h(u) \rightarrow 0$ faster than any power law as $u \rightarrow \infty$. A second integration then gives $h(u) \propto \exp \left(-u^{4 / 3}\right)$, from which we conclude that the longitudinal probability distribution has the form

$$
P(x, t) \propto t^{-3 / 4} \exp \left(-\left(x / t^{3 / 4}\right)^{4 / 3}\right) .
$$

This functional form coincides with that obtained previously by a different method [2,3] in which the dominant contribution to the large- $u$ tail of $P(x, t)$ arises from extreme "stretched" trajectories in unlikely velocity configurations. Thus the observation of the large- $u$ tail for $P(x, t)$ from the numerical data in Fig. 5 can again be anticipated to be problematical; much more extensive simulation would be needed.

\section{DISCUSSION}

We have investigated the time dependence of the configuration averaged survival probability, $S(t)$, in a semiinfinite two-dimensional system for diffusing particles which are also driven by a unidirectional random zeromean velocity field, $v_{x}(y)$. A qualitative argument suggests that $S(t) \propto t^{-1 / 4}$, a prediction which is in excellent agreement with numerical results. We also examined the longitudinal spatial probability density of the surviving particles, $P(x, t)$. Interestingly, although the numerical evidence supporting the prediction that $S(t) \propto t^{-1 / 4}$ is strong, the numerical data for $P(x, t)$ indicates slow convergence to the scaling limit and some inconsistency with the behavior of $S(t)$ itself. Similar anomalies in the probability distribution occur in the unbounded geometry [2], due to the contribution of extreme velocity 
configurations in the average. It is surprising that the behavior of $S(t)$ is apparently relatively insensitive to the contribution of such extreme configurations.

An interesting byproduct of the spatial distribution of the surviving particles is that the form $\frac{-\partial P(x, t)}{\partial x^{1 / 3}}$ is suggested as the appropriate expression for the particle flux. This leads to a scale dependent diffusion coefficient which is proportion to $x^{2 / 3}$, as well as the continuum equation of motion, Eq. (11), for the longitudinal spatial probability distribution in an unbounded geometry. The solution to this equation of motion is simple to obtain and reproduces the known asymptotic form of $P(x, t)$ in the unbounded geometry [2]. As an application of this equation of motion, we find new predictions for steady-state transport properties. For example, for a steady input of particles at $x=L$ and particle absorption at $x=0$, the steady solution to Eq. (11) gives a configuration-averaged density profile which varies as $x^{1 / 3}$. It will be worthwhile to test this prediction and also the general prescription for obtaining an effective equation of motion from the behavior of the particle flux near an absorbing boundary.

Finally, the behavior of $S(t)$ for a semi-infinite system with a longitudinal MdM velocity field can be easily generalized to arbitrary spatial dimension $d$. From classical results [5], the number of distinct longitudinal rows visited by a random walk in time $t$ varies as $t^{(d-1) / 2}$ for dimension $2<d<3$ (i. e., transverse spatial dimension between 1 and 2 ), as $t / \ln t$ for $d=3$, and as $t$ for $d>3$. Following closely the approach in Sec. II, this then leads to

$$
S(t) \sim \begin{cases}t^{-1 / 4} & d=2 ; \\ t^{-(d-1) / 4} & 2<d<3 \\ (\ln t / t)^{1 / 2} & d=3 ; \\ t^{-1 / 2} & d>3 .\end{cases}
$$

Thus above three dimensions, the survival probability exponent value is not affected by the presence of a random velocity field.

I thank P. L. Krapivsky for many helpful discussions and a critical reading of the manuscript. This research was supported in part by the NSF grant number DMR9632059. This financial assistance is gratefully acknowledged.

[1] G. Matheron and G. de Marsily, Water Resources Res. 16, 901 (1980).

[2] J.-P. Bouchaud, A. Georges, J. Koplik, A. Provata, and S. Redner, Phys. Rev. Lett. 64, 2503 (1990); J.P. Bouchaud and A. Georges, Phys. Reports 195, 127 (1990).

[3] S. Redner, Physica A 168, 551 (1990).

[4] G. Zumofen, J. Klafter, and A. Blumen Phys. Rev. A 42, 4601 (1990).

[5] See, e. g., G. H. Weiss and R. J. Rubin, Adv. Chem. Phys. 52, 363 (1983), and references therein.

[6] S. Redner and P. L. Krapivsky, J. Stat. Phys. 82, 999 (1996).

[7] See e. g., S. Havlin and D. ben-Avraham. Adv. Phys. 36, 695 (1987).

[8] G. I. Taylor, Proc. Roy. Soc. London, Ser. A 219, 186 (1953). 